\title{
Interplay between Ubiquitin, SUMO, and Poly(ADP-Ribose) in the Cellular Response to Genotoxic Stress
}

\author{
Stefania Pellegrino and Matthias Altmeyer* \\ Department of Molecular Mechanisms of Disease, University of Zurich, Zürich, Switzerland
}

Cells employ a complex network of molecular pathways to cope with endogenous and exogenous genotoxic stress. This multilayered response ensures that genomic lesions are efficiently detected and faithfully repaired in order to safeguard genome integrity. The molecular choreography at sites of DNA damage relies heavily on post-translational modifications (PTMs). Protein modifications with ubiquitin and the small ubiquitin-like modifier SUMO have recently emerged as important regulatory means to coordinate DNA damage signaling and repair. Both ubiquitylation and SUMOylation can lead to extensive chain-like protein modifications, a feature that is shared with yet another DNA damage-induced PTM, the modification of proteins with poly(ADP-ribose) (PAR). Chains of ubiquitin, SUMO, and PAR all contribute to the multi-protein assemblies found at sites

OPEN ACCESS

Edited by:

Kristijan Ramadan,

University of Oxford, UK

Reviewed by:

Nico P. Dantuma,

Karolinska Institutet, Sweden Howard Donninger

University of Louisville, USA

*Correspondence:

Matthias Altmeyer matthias.altmeyer@uzh.ch

Specialty section: This article was submitted to Cancer Genetics,

a section of the journal

Frontiers in Genetics

Received: 28 February 2016 Accepted: 04 April 2016 Published: 19 April 2016

Citation:

Pellegrino S and Altmeyer M (2016) Interplay between Ubiquitin, SUMO, and Poly(ADP-Ribose) in the Cellular

Response to Genotoxic Stress.

Front. Genet. 7:63.

doi: 10.3389/fgene.2016.00063 of DNA damage and regulate their spatio-temporal dynamics. Here, we review recent advancements in our understanding of how ubiquitin, SUMO, and PAR coordinate the DNA damage response and highlight emerging examples of an intricate interplay between these chain-like modifications during the cellular response to genotoxic stress.

Keywords: ubiquitin, SUMO, poly(ADP-ribose), PARP, DNA damage response, DDR, genome stability, cancer

\section{INTRODUCTION}

Our genetic material is under constant cellular surveillance and care. Maintaining genome stability is indeed a vital task, not only under conditions when external toxins or physical strains challenge the integrity of the genome, but also in the course of normal cellular metabolism, when reactive metabolites and physiological DNA transactions can lead to a plethora of lesions. If these damages are not detected and faithfully repaired, cells run the risk of accumulating mutations that can erode genome function, vitiate cell fate, or compromise cell survival. Faced with such threats cells have developed sophisticated mechanisms to sense and repair damaged DNA. These mechanisms, which are collectively referred to as the DNA damage response (DDR), not only ensure that most lesions are efficiently repaired, but they also coordinate genome integrity maintenance with other cellular functions such as transcription, DNA replication, and cell cycle progression (Ciccia and Elledge, 2010). The DDR is an intricate molecular network that safeguards genome integrity and helps to maintain cell identity, thus constituting a natural barrier against the development of various human diseases (Jackson and Bartek, 2009). Underpinning the crucial role of genome integrity maintenance for human health, a deteriorated DDR and signs of genome instability are typical features of many human cancers, and they represent cancer-specific vulnerabilities that can be targeted by precision therapies (O'Connor, 2015). 
To fulfill its task, the DDR employs a multitude of tightly regulated posttranslational protein modifications (PTMs). In addition to modulating protein functions locally at the damage site, PTMs play important roles in spreading the DNA damage signal to the surrounding chromatin (Lukas et al., 2011; Polo and Jackson, 2011) and in activating cell cycle checkpoints (Stracker et al., 2009). Positive feedback mechanisms amplify the DNA damage signal and enable sustained accumulation of genome caretaker proteins, while antagonistic mechanisms ensure that modifications induced by DNA damage remain spatially and temporally confined (van Attikum and Gasser, 2009; Altmeyer and Lukas, 2013a,b; Panier and Durocher, 2013). Multiple PTMs cooperate in this spatio-temporal regulation and can either act in series, in parallel or in a combinatorial fashion to dynamically reshape the chromatin landscape around DNA lesions and prepare the stage for repair (Dantuma and van Attikum, 2016). This barcoding involves multi-target phosphorylation (Marechal and Zou, 2013; Shiloh and Ziv, 2013; Davis et al., 2014; Awasthi et al., 2015; Paull, 2015), as well as acetylation and methylation (Gong and Miller, 2013; Hendzel and Greenberg, 2013; Price and D'Andrea, 2013). In addition to these small moiety modifications, recent work revealed how larger PTMs, which can form extensive modification chains, coordinate the access of genome caretakers to DNA lesions and regulate repair pathway choices. Here, we briefly discuss how ubiquitylation, SUMOylation, and poly(ADPribosyl)ation (PARylation) are employed by the DDR, before we highlight emerging examples that have started to elucidate an intricate and still incompletely understood crosstalk between these catenarian modifications in response to DNA damage. We focus our analysis primarily on the response of mammalian cells to DNA double strand breaks (DSBs), yet an equally well-coordinated crosstalk between chromatin-based PTMs also operates in other situations of genotoxic stress (Kim and D’Andrea, 2012; Marteijn et al., 2014; Ulrich, 2014).

\section{UBIQUITIN CONJUGATION AROUND DSB SITES}

Chromosome breaks are among the most toxic DNA lesions and two major repair pathways evolved to deal with DSBs. The non-homologous end-joining (NHEJ) pathway is independent of intact template DNA sequences and can re-ligate broken DNA ends throughout the cell cycle. In contrast, faithful repair by homologous recombination (HR) depends on an undamaged template DNA and is thus restricted to the S/G2 phases of the cell cycle when sister chromatids are available. While NHEJ is generally considered error-prone due to the risk of nucleotide loss from DNA ends, HR is considered to be more accurate due to template-based repair. The choice between NHEJ and HR is tightly controlled, and imbalances in its regulation can lead to genome instability and accelerate cancer development (Chapman et al., 2012; Aparicio et al., 2014). Interestingly, the recruitment of several key repair pathway choice mediators to DNA break sites depends on local ubiquitin conjugations (Messick and Greenberg, 2009; Pinder et al., 2013). Indeed, one of the central players of repair pathway choice is the ubiquitin-sensing genome caretaker protein 53BP1, whose recruitment to DSBs requires the consecutive action of the ubiquitin E3 ligases RNF8 and RNF168 (Panier and Boulton, 2014). In a concerted manner, and initiated by upstream phosphorylation of the histone variant H2AX, RNF8, and RNF168 ubiquitylate histones $\mathrm{H} 1$ and H2A, respectively, and thereby provide a landing platform for 53BP1 (Mattiroli et al., 2012; Fradet-Turcotte et al., 2013; Gatti et al., 2015; Thorslund et al., 2015). 53BP1 in turn assembles the effector proteins RIF1, PTIP, Artemis, and MAD2L2/REV7 to limit the extent of DNA end resection and thereby channel repair toward NHEJ (Figure 1A) (Callen et al., 2013; Chapman et al., 2013; Di Virgilio et al., 2013; Escribano-Diaz et al., 2013; Zimmermann et al., 2013; Wang et al., 2014; Boersma et al., 2015; Xu et al., 2015). Of note, the functions of 53BP1 and its effectors are required for the hypersensitivity of HR-defective cancer cells to inhibitors of PAR polymerases (Lord and Ashworth, 2016), thus linking the consequences of compromised PARylation to the effects of a ubiquitin-dependent anti-resection barrier under pathological repair pathway choice conditions.

Importantly, ubiquitin conjugation is not only involved in generating the ubiquitin code that is recognized by 53BP1, but also fosters 53BP1 accumulation by RNF8/RNF168-dependent and $\mathrm{VCP} / \mathrm{p} 97$-mediated removal of proteins from damaged chromatin (Acs et al., 2011; Meerang et al., 2011). As was shown for the H4K20me2-binding proteins L3MBTL1 and JMJD2A/KDM4A, this can unmask additional binding sites for the tandem tudor domain of 53BP1 (Acs et al., 2011; Mallette et al., 2012). Thus, the removal of chromatin binders seems to converge with the generation of new chromatin marks to allow for the efficient recruitment of 53BP1 and its downstream effectors (Figure 1A).

While these reactions build up an important anti-resection barrier that shields broken DNA ends from unscheduled nucleolytic digestion, ubiquitin conjugation also plays a role in promoting HR. For example, the ubiquitin E3 ligases TRIP12 and UBR5 cooperate to keep RNF168 levels in check and thereby prevent excessive 53BP1 function (Gudjonsson et al., 2012). More recently, the ubiquitin E3 ligase RNF138 was shown to accumulate at sites of DNA damage where it stimulates DNA end resection and promotes HR (Ismail et al., 2015; Schmidt et al., 2015). Thus, rather than channeling repair pathway choices in one direction, the ubiquitylation system employs rheostats and antagonistic sub-pathways to regulate repair decisions in a manner that likely integrates information about cell cycle phase and chromatin context.

\section{SUMO CONJUGATION AT DSB SITES ASSISTS THE DDR}

Just like ubiquitylation, also SUMOylation plays important roles for the tightly controlled protein choreography at DSB sites, and its deregulation impairs genome stability and cell proliferation (Bekker-Jensen and Mailand, 2011; Jackson and Durocher, 2013; Eifler and Vertegaal, 2015). The SUMO isoforms SUMO1 and SUMO2/3 were all found to accumulate at sites of DNA damage in a manner dependent on the SUMO E3 ligases 
A

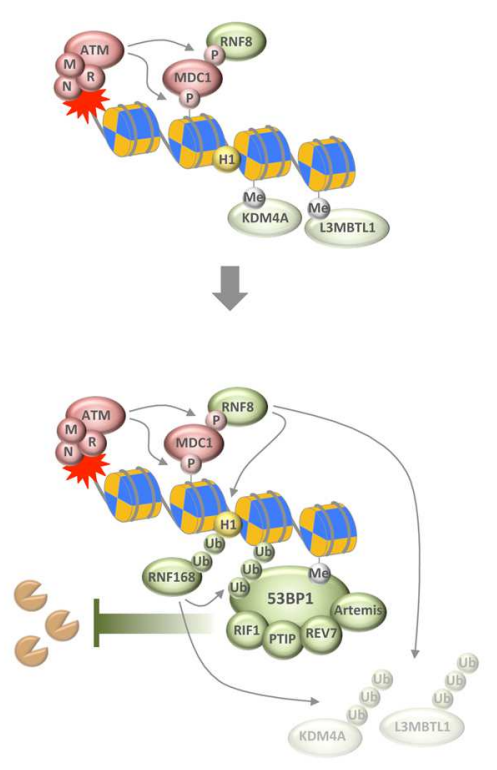

B

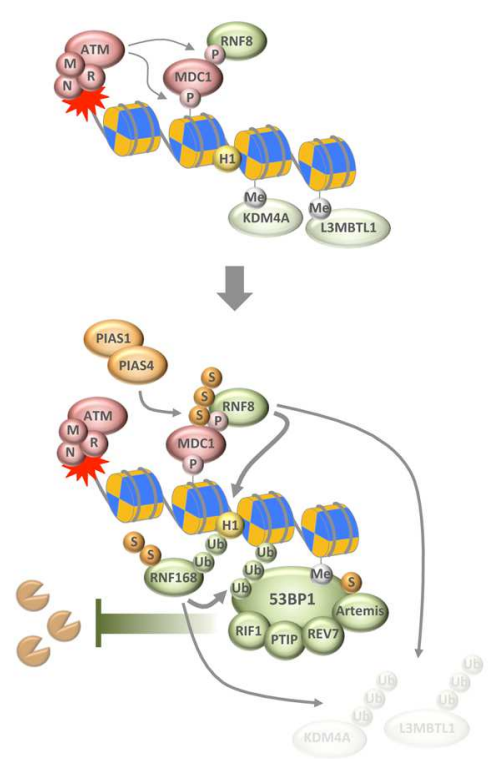

C
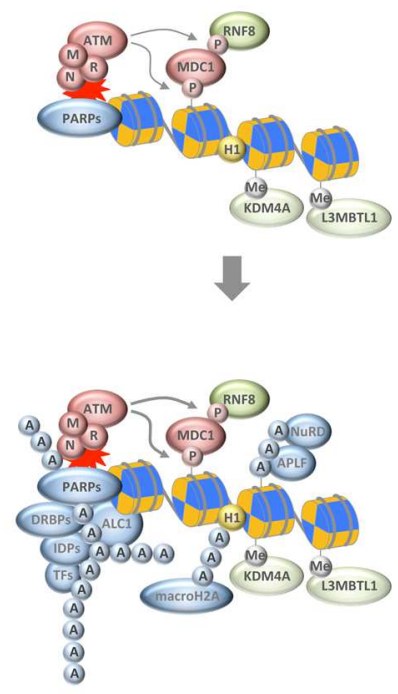

FIGURE 1 | Chain-like modifications build up dynamic DNA repair compartments that orchestrate the DNA damage response (DDR). (A) In response to DNA damage, and subsequent to the MRN/ATM/MDC1-driven phosphorylation of histone variant H2AX, ubiquitylation of $\mathrm{H} 1$ and $\mathrm{H} 2 \mathrm{~B}$ by RNF8 and RNF168, respectively, synergizes with ubiquitin-dependent extraction of proteins from the damaged chromatin to promote the recruitment of 53BP1 and its downstream effectors. (B) SUMOylation by PIAS1 and PIAS4 further enhances ubiquitin conjugation around DNA break sites. (C) Poly(ADP-ribosyl)ation (PARylation) by PARPs generates a recruitment platform for a plethora of PAR-binding proteins, including various transcription factors (TFs), DNA- and RNA-binding proteins (DRBPs), and a set of intrinsically disordered proteins (IDPs). P, phosphorylation; Me, methylation; Ub, ubiquitylation; S, SUMOylation; A, ADP-ribosylation; M, MRE11; R, RAD50; N, NBS1.

PIAS1 and PIAS4 (Galanty et al., 2009; Morris et al., 2009). Interestingly, the SUMO conjugation observed in response to DNA breakage promotes accumulation of ubiquitin chains on damaged chromatin and is required for the efficient recruitment of ubiquitin-dependent genome caretakers (Galanty et al., 2009; Morris et al., 2009). Among the targets of DNA damage-induced SUMOylation are MDC1, RNF168, 53BP1, BRCA1, RPA, and EXO1 (Figure 1B) (Galanty et al., 2009; Morris et al., 2009; Luo et al., 2012; Yin et al., 2012; Bologna et al., 2015; Hendriks et al., 2015). SUMOylation not only contributes to the recruitment of proteins to DSBs but also to their coordinated removal, and, interestingly, is required for both NHEJ and HR (Galanty et al., 2009; Morris et al., 2009; Chung and Zhao, 2015; Sarangi et al., 2015). While much remains to be learnt about the exact mechanisms how SUMOylation and SUMO chain formation in particular affect the repair of DSBs, it has become clear that the SUMOylation and ubiquitylation machineries work closely together to help restore genome integrity upon chromosome breakage (see below).

\section{POLY(ADP-RIBOSE) CHAINS ATTRACT A DIVERSE SET OF PROTEINS TO DNA BREAK SITES}

A third type of protein modification that comes in chains and ties proteins to DNA breaks sites is PARylation. Catalyzed by PAR polymerases (PARPs) in response to genotoxic stress, DNA breakassociated ADP-ribose polymers provide a landing platform for a plethora of PAR-binding proteins (Teloni and Altmeyer, 2016). This includes chromatin remodelers and DNA repair factors, but also proteins involved in nucleic acid metabolism and RNA processing (Krietsch et al., 2013; Golia et al., 2015; Izhar et al., 2015; Teloni and Altmeyer, 2016). PAR-dependent events have been implicated in the cellular response to DNA single-strand breaks and in maintaining the integrity of perturbed replication forks, but also contribute to DSB repair (Beck et al., 2014). Among the proteins that respond to PAR formation are the DDR factors MRE11 and NBS1 (Haince et al., 2008), the chromatin remodeler ALC1 (Ahel et al., 2009; Gottschalk et al., 2009), the histone variant macroH2A (Timinszky et al., 2009), components of the repressive polycomb and NuRD complexes (Chou et al., 2010; Polo et al., 2010), NHEJ and HR factors (Ahel et al., 2008; Rulten et al., 2008; Li and Yu, 2013; Zhang et al., 2015), and a class of intrinsically disordered proteins that can phase separate to generate dynamic compartments (Figure 1C) (Altmeyer et al., 2015; Patel et al., 2015). The relative contribution of each of these recruitments for faithful DNA repair is insufficiently understood and may depend on the type of damage and its complexity as well as the overall damage load. The amount and type of damage, together with cell cycle phase and local chromatin environment, are likely to influence the number of PAR chains generated, their length and branching frequency, and may thereby impact on the protein recruitments that are driven by PAR formation. 
Given that all three catenarian modifications, ubiquitylation, SUMOylation, and PARylation, orchestrate the protein accumulations around DNA break sites, significant crosstalk exists. In the following paragraphs, we highlight emerging examples of such interplay and how it affects the accrual of genome caretakers at damaged chromatin.

\section{INTERPLAY BETWEEN UBIQUITIN AND SUMO}

As noted above, the ubiquitylation and SUMOylation machineries are tightly interconnected and cooperate to reshape the chromatin landscape for proper repair (BekkerJensen and Mailand, 2011; Jackson and Durocher, 2013). An interesting direct link between the two systems is provided by SUMO-targeted ubiquitin ligases (STUbLs), readers of SUMO modifications that possess ubiquitin ligase activity and specifically modify SUMOylated substrates. The STUbL RNF4 is a prime example that recently emerged as important regulator of protein accumulation upon DNA breakage. RNF4 is recruited to DSBs via its SUMO interaction motifs and ubiquitylates SUMOylated DDR factors, thereby leading to their withdrawal from repair sites and initiating their proteasomal degradation (Galanty et al., 2012; Luo et al., 2012; Yin et al., 2012). Defective targeting by RNF4 enhances the retention of a subset of DDR factors and compromises the initiation of downstream events required for efficient repair. Among the proteins that are targeted by RNF4 is the adaptor protein MDC1, whose removal promotes access of the DNA end resection and HR machineries (Galanty et al., 2012; Luo et al., 2012; Yin et al., 2012). Once DNA end resection has occurred, RNF4 is again required for the extraction of the single-stranded DNA binding protein RPA, which in turn allows for the accumulation of BRCA2 and RAD51 on resected DNA (Galanty et al., 2012). Collectively, these findings suggest that SUMO-targeted ubiquitylation participates in the dismantling of the anti-resection barrier and promotes HR reactions. In support of this notion, the activity of RNF4 itself is regulated in a CDK-dependent manner, allowing it to fulfill its HR-promoting roles primarily in the S/G2 phases of the cell cycle (Figure 2A) (Luo et al., 2015; Kuo et al., 2016). Interestingly, the DNA damage-induced crosstalk between SUMOylation and ubiquitylation is not restricted to DSBs (Poulsen et al., 2013; Ragland et al., 2013; Gibbs-Seymour et al., 2015; van Cuijk et al., 2015), and SUMO-targeted ubiquitylation followed by targeted protein removal and/or degradation thus emerges as a common theme in the stepwise progression of DNA repair pathways.

\section{INTERPLAY BETWEEN POLY(ADP-RIBOSE) AND UBIQUITIN}

In analogy to SUMO-targeted ubiquitylation by STUbLs it was recently discovered that also PAR serves as recognition signal for selected ubiquitin ligases (Zhang et al., 2011; Zhou et al., 2011; Wang et al., 2012). The best-characterized PAR-targeted ubiquitin ligase (PTUbL) is RNF146/Iduna. By virtue of its PAR-binding WWE domain RNF146 is recruited to PARylated proteins where the WWE-PAR interaction leads to an allosteric activation of its ubiquitin ligase domain (DaRosa et al., 2015). Among the proteins that are ubiquitylated by RNF146 are PARP1, PARP2, KU70, DNA ligase III, and XRCC1 (Kang et al., 2011).
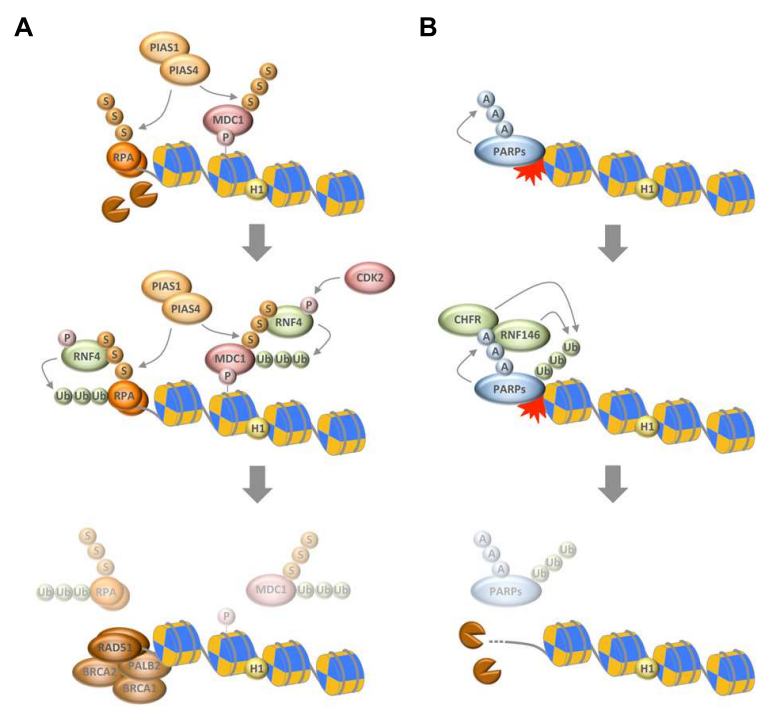

C
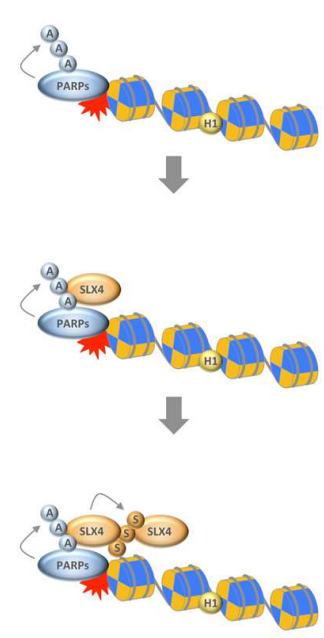
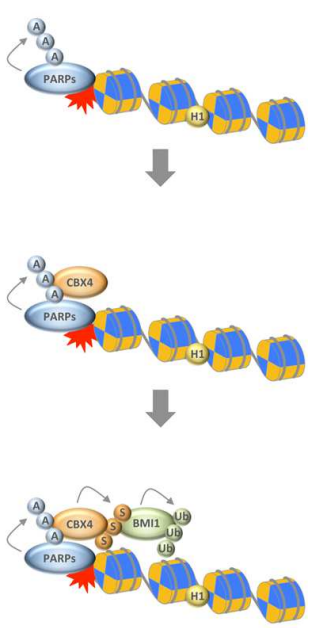

FIGURE 2 | Interplay between chain-like modifications at sites of DNA damage. (A) Interplay between ubiquitylation and SUMOylation through the STUbL RNF4, which ubiquitylates SUMOylated substrates to mediate their timely removal from repair sites. (B) Interplay between PARylation and ubiquitylation through the PTUbLs RNF146 and CHFR, which cooperate to dissociate automodified PARP1 from DNA break sites. (C) Productive interaction between PARylation and SUMOylation to stabilize the recruitment of the SLX4 complex. (D) Interplay between PARylation, SUMOylation, and ubiquitylation via the PAR-responsive SUMO ligase CBX4 and the SUMO-responsive ubiquitin ligase BMl1 to promote chromatin ubiquitylation in response to DNA damage. P, phosphorylation; Ub, ubiquitylation; S, SUMOylation; A, ADP-ribosylation. 
Thus, and in parallel to DNA break-induced SUMOylation, also PAR participates in the targeted protein ubiquitylation and turnover at sites of genomic lesions.

While these events likely evolved to prevent excessive interactions of repair factors with DNA break sites, PARdependent ubiquitylation also assists the early recruitment of genome caretakers. For instance, PAR formation was shown to be required for the recruitment of the BAL1/BBAP ubiquitin ligase complex, whose activity promotes the retention of the RAP80BRCA1 complex (Yan et al., 2013). This mechanism seems to act in parallel to the PAR-mediated recruitment of BRCA1 via the PAR-binding BRCT domains of its partner protein BARD1 (Li and Yu, 2013), and is part of the PAR-dependent selective interaction filtering that is observed almost immediately upon DNA damage induction and temporally precedes the full buildup of the RNF8/RNF168-dependent ubiquitin compartment (Altmeyer et al., 2015; Teloni and Altmeyer, 2016). Notably, even at later stages of the chromatin response to DNA damage interplay between PARylation and ubiquitylation seems to exist, because the PAR-dependent recruitment of the chromatin remodeler SMARCA5/SNF2H facilitates RNF168 accumulation and promotes efficient ubiquitin conjugation (Smeenk et al., 2013).

Another RING-type ubiquitin E3 ligase, whose role in genome integrity maintenance is linked to PAR formation, is the mitotic regulator CHFR. A PAR-binding zinc finger motif (PBZ) mediates its interaction with genotoxic stress-induced PAR and was shown to be required for the CHFR-dependent antephase checkpoint (Ahel et al., 2008; Oberoi et al., 2010). Interestingly, the functions of CHFR also feed into histone acetylation and ATM activation (Wu et al., 2011), and mediate the first wave of ubiquitylation in response to DNA damage (Liu et al., 2013). As part of this response, CHFR ubiquitylates PARP1 itself, leading to its dissociation from DNA break sites, thus representing another example of PTUbL-mediated stepwise succession of repair events (Kashima et al., 2012; Liu et al., 2013).

Taken together, PARylation assists the early recruitment of genome caretakers, including various ubiquitin ligases, which further promote chromatin modifications and lead to the formation of a dedicated repair compartment, but it also participates in temporarily restraining protein access to repair sites and in the timely and PTUbL-mediated removal of repair factors once they have fulfilled their duties (Figure 2B).

\section{INTERPLAY BETWEEN POLY(ADP-RIBOSE) AND SUMO}

The first direct links between PARylation and SUMOylation were described in the context of PARP1-regulated transcription (Martin et al., 2009; Messner et al., 2009), and SUMOylation of PARP1 was indeed found to be largely irresponsive to DNA damage (Zilio et al., 2013). More recently, however, a functional crosstalk between these two chain-like modifications has started to emerge also in the context of genome integrity maintenance. For instance, PARylation of tyrosyl-DNA phosphodiesterase 1 (TDP1) was shown to cooperate with TDP1 SUMOylation to stabilize the protein and promote its function in the repair of trapped topoisomerase I (TOP1) cleavage complexes (Das et al., 2014). Similarly, PARylation and SUMOylation cooperate to recruit and stabilize the SLX4 nuclease scaffold complex, itself a SUMO E3 ligase, at DNA damage sites (Figure 2C) (Gonzalez-Prieto et al., 2015; Guervilly et al., 2015). Finally, and as an example for productive interplay between all three chainlike modifications, PARylation is required for the recruitment of CBX4. In a pathway that functions in parallel to the PIAS1/PIAS4-mediated SUMOylation at damaged chromatin, PAR-dependent SUMOylation by CBX4 attracts the polycomb ubiquitin E3 ligase BMI1, which in turn contributes to DNA damage-induced histone ubiquitylation and promotes repair (Figure 2D) (Ismail et al., 2010, 2012, 2015; Ginjala et al., 2011). Thus, intriguing examples of close cooperation between catenarian modifications exist, and future findings are likely to shed more light onto the intricate interplay between ubiquitin, SUMO and PAR in the DDR.

\section{CONCLUSION AND OUTLOOK}

While distinct in their chemical nature and regulatory mechanism, ubiquitylation, SUMOylation, and PARylation share the feature of being chain-like protein modifications. The composition of modification chains, their length, linkage type and branching frequency contains information that can be used by complex regulatory circuits and signaling pathways such as the DDR. Recent work has elucidated how cells employ a sophisticated sequence of reactions with remarkable temporal and spatial resolution to shield genomic lesions and build up dynamic functional platforms that promote repair. The information content imbedded in this response is immense, and the use of modification chains may thus support the need for lesion-specific chromatin barcodes that dynamically change as repair reactions progress.

The multivalent protein recruitment polymers formed by ubiquitylation, SUMOylation, and PARylation often cooperate to achieve robust responses. To this end, they act successively or in parallel, and frequently use positive feedback loops to amplify the signal and increase its specificity. They also employ timedelayed negative feedback to terminate reactions and disassemble complexes, which are no longer needed and constitute roadblocks for downstream events. While recent work has started to elucidate the crosstalk between these modifications, how their combinatorial use and dynamic interplay reshapes the chromatin environment surrounding different types of genomic lesions, dictates repair pathway decisions, and determines repair fidelity remains incompletely understood. Moreover, almost nothing is known about mixed chain modifications, e.g., PARylation of ubiquitin or SUMO chains, and how they might be employed by the DDR. Quantitative time-resolved proteomics and imaging approaches that provide spatial information about protein redistribution and can relate this to cell cycle information are powerful tools to address these issues. The insights gained will not only deepen our understanding of the DDR, but may also provide additional clues to the mechanisms that underlie 
the toxicity of inhibiting chain-like modifications in cancer treatments.

\section{AUTHOR CONTRIBUTIONS}

All authors listed, have made substantial, direct and intellectual contribution to the work, and approved it for publication.

\section{FUNDING}

Research in the lab of MA is supported by the Swiss National Science Foundation (SNSF Professorship Grant PP00P3_150690)

\section{REFERENCES}

Acs, K., Luijsterburg, M. S., Ackermann, L., Salomons, F. A., Hoppe, T., and Dantuma, N. P. (2011). The AAA-ATPase VCP/p97 promotes 53BP1 recruitment by removing L3MBTL1 from DNA double-strand breaks. Nat. Struct. Mol. Biol. 18, 1345-1350. doi: 10.1038/nsmb.2188

Ahel, D., Horejsi, Z., Wiechens, N., Polo, S. E., Garcia-Wilson, E., Ahel, I., et al. (2009). Poly(ADP-ribose)-dependent regulation of DNA repair by the chromatin remodeling enzyme ALC1. Science 325, 1240-1243. doi: $10.1126 /$ science. 1177321

Ahel, I., Ahel, D., Matsusaka, T., Clark, A. J., Pines, J., Boulton, S. J., et al. (2008). Poly(ADP-ribose)-binding zinc finger motifs in DNA repair/checkpoint proteins. Nature 451, 81-85. doi: 10.1038/nature 06420

Altmeyer, M., and Lukas, J. (2013a). Guarding against collateral damage during chromatin transactions. Cell 153, 1431-1434. doi: 10.1016/j.cell.2013.05.044

Altmeyer, M., and Lukas, J. (2013b). To spread or not to spread-chromatin modifications in response to DNA damage. Curr. Opin. Genet. Dev. 23, 156-165. doi: 10.1016/j.gde.2012.11.001

Altmeyer, M., Neelsen, K. J., Teloni, F., Pozdnyakova, I., Pellegrino, S., Grofte, M., et al. (2015). Liquid demixing of intrinsically disordered proteins is seeded by poly(ADP-ribose). Nat. Commun. 6, 8088. doi: 10.1038/ncomms9088

Aparicio, T., Baer, R., and Gautier, J. (2014). DNA double-strand break repair pathway choice and cancer. DNA Repair (Amst.) 19, 169-175. doi: 10.1016/j.dnarep.2014.03.014

Awasthi, P., Foiani, M., and Kumar, A. (2015). ATM and ATR signaling at a glance. J. Cell Sci. 128, 4255-4262. doi: 10.1242/jcs. 169730

Beck, C., Robert, I., Reina-San-Martin, B., Schreiber, V., and Dantzer, F. (2014). Poly(ADP-ribose) polymerases in double-strand break repair: focus on PARP1, PARP2 and PARP3. Exp. Cell Res. 329, 18-25. doi: 10.1016/j.yexcr.2014.07.003

Bekker-Jensen, S., and Mailand, N. (2011). The ubiquitin- and SUMO-dependent signaling response to DNA double-strand breaks. FEBS Lett. 585, 2914-2919. doi: 10.1016/j.febslet.2011.05.056

Boersma, V., Moatti, N., Segura-Bayona, S., Peuscher, M. H., van der Torre, J., Wevers, B. A., et al. (2015). MAD2L2 controls DNA repair at telomeres and DNA breaks by inhibiting $5^{\prime}$ end resection. Nature 521, 537-540. doi: 10.1038 /nature 14216

Bologna, S., Altmannova, V., Valtorta, E., Koenig, C., Liberali, P., Gentili, C., et al. (2015). Sumoylation regulates EXO1 stability and processing of DNA damage. Cell Cycle 14, 2439-2450. doi: 10.1080/15384101.2015.1060381

Callen, E., Di Virgilio, M., Kruhlak, M. J., Nieto-Soler, M., Wong, N., Chen, H. T., et al. (2013). 53BP1 mediates productive and mutagenic DNA repair through distinct phosphoprotein interactions. Cell 153, 1266-1280. doi: 10.1016/j.cell.2013.05.023

Chapman, J. R., Barral, P., Vannier, J. B., Borel, V., Steger, M., Tomas-Loba, A., et al. (2013). RIF1 is essential for 53BP1-dependent nonhomologous end joining and suppression of DNA double-strand break resection. Mol. Cell 49, 858-871. doi: 10.1016/j.molcel.2013.01.002

Chapman, J. R., Taylor, M. R., and Boulton, S. J. (2012). Playing the end game: DNA double-strand break repair pathway choice. Mol. Cell 47, 497-510. doi: 10.1016/j.molcel.2012.07.029 and by the University of Zurich Association Research Talent Development Fund.

\section{ACKNOWLEDGMENTS}

We apologize to authors whose work could not be cited due to space limitations. We are thankful to all members of the Altmeyer Lab and of the Department of Molecular Mechanisms of Disease for helpful discussions, and acknowledge support from the Molecular Life Sciences Program of the Life Science Zurich Graduate School.

Chou, D. M., Adamson, B., Dephoure, N. E., Tan, X., Nottke, A. C., Hurov, K. E., et al. (2010). A chromatin localization screen reveals poly (ADP ribose)regulated recruitment of the repressive polycomb and NuRD complexes to sites of DNA damage. Proc. Natl. Acad. Sci. U.S.A. 107, 18475-18480. doi: 10.1073/pnas.1012946107

Chung, I., and Zhao, X. (2015). DNA break-induced sumoylation is enabled by collaboration between a SUMO ligase and the ssDNA-binding complex RPA. Genes Dev. 29, 1593-1598. doi: 10.1101/gad.265058.115

Ciccia, A., and Elledge, S. J. (2010). The DNA damage response: making it safe to play with knives. Mol. Cell 40, 179-204. doi: 10.1016/j.molcel.2010.09.019

Dantuma, N. P., and van Attikum, H. (2016). Spatiotemporal regulation of posttranslational modifications in the DNA damage response. EMBO J. 35, 6-23. doi: 10.15252/embj.201592595

DaRosa, P. A., Wang, Z., Jiang, X., Pruneda, J. N., Cong, F., Klevit, R. E., et al. (2015). Allosteric activation of the RNF146 ubiquitin ligase by a poly(ADPribosyl)ation signal. Nature 517, 223-226. doi: 10.1038/nature13826

Das, B. B., Huang, S. Y., Murai, J., Rehman, I., Ame, J. C., Sengupta, S., et al. (2014). PARP1-TDP1 coupling for the repair of topoisomerase I-induced DNA damage. Nucleic Acids Res. 42, 4435-4449. doi: 10.1093/nar/gku088

Davis, A. J., Chen, B. P. C., and Chen, D. J. (2014). DNA-PK: a dynamic enzyme in a versatile DSB repair pathway. DNA Repair 17, 21-29. doi: 10.1016/j.dnarep.2014.02.020

Di Virgilio, M., Callen, E., Yamane, A., Zhang, W., Jankovic, M., Gitlin, A. D., et al. (2013). Rif1 prevents resection of DNA breaks and promotes immunoglobulin class switching. Science 339, 711-715. doi: 10.1126/science.1230624

Eifler, K., and Vertegaal, A. C. (2015). SUMOylation-mediated regulation of cell cycle progression and cancer. Trends Biochem. Sci. 40, 779-793. doi: 10.1016/j.tibs.2015.09.006

Escribano-Diaz, C., Orthwein, A., Fradet-Turcotte, A., Xing, M., Young, J. T., Tkac, J., et al. (2013). A cell cycle-dependent regulatory circuit composed of 53BP1-RIF1 and BRCA1-CtIP controls DNA repair pathway choice. Mol. Cell 49, 872-883. doi: 10.1016/j.molcel.2013.01.001

Fradet-Turcotte, A., Canny, M. D., Escribano-Diaz, C., Orthwein, A., Leung, C. C. Y., Huang, H., et al. (2013). 53BP1 is a reader of the DNA-damageinduced H2A Lys 15 ubiquitin mark. Nature 499, 50-54. doi: 10.1038/nature 12318

Galanty, Y., Belotserkovskaya, R., Coates, J., and Jackson, S. P. (2012). RNF4, a SUMO-targeted ubiquitin E3 ligase, promotes DNA double-strand break repair. Genes Dev. 26, 1179-1195. doi: 10.1101/gad.188284.112

Galanty, Y., Belotserkovskaya, R., Coates, J., Polo, S., Miller, K. M., and Jackson, S. P. (2009). Mammalian SUMO E3-ligases PIAS1 and PIAS4 promote responses to DNA double-strand breaks. Nature 462, 935-939. doi: 10.1038/nature08657

Gatti, M., Pinato, S., Maiolica, A., Rocchio, F., Prato, M. G., Aebersold, R., et al. (2015). RNF168 promotes noncanonical K27 ubiquitination to signal DNA damage. Cell Rep. 10, 226-238. doi: 10.1016/j.celrep.2014.12.021

Gibbs-Seymour, I., Oka, Y., Rajendra, E., Weinert, B. T., Passmore, L. A., Patel, K. J., et al. (2015). Ubiquitin-SUMO circuitry controls activated fanconi anemia ID complex dosage in response to DNA damage. Mol. Cell 57, 150-164. doi: 10.1016/j.molcel.2014.12.001 
Ginjala, V., Nacerddine, K., Kulkarni, A., Oza, J., Hill, S. J., Yao, M., et al. (2011). BMI1 Is recruited to DNA breaks and contributes to DNA damageinduced H2A ubiquitination and repair. Mol. Cell. Biol. 31, 1972-1982. doi: 10.1128/Mcb.00981-10

Golia, B., Singh, H. R., and Timinszky, G. (2015). Poly-ADP-ribosylation signaling during DNA damage repair. Front. Biosci. 20:440-457. doi: 10.2741/4318

Gong, F. D., and Miller, K. M. (2013). Mammalian DNA repair: HATs and HDACs make their mark through histone acetylation. Mutat. Res. 750, 23-30. doi: 10.1016/j.mrfmmm.2013.07.002

Gonzalez-Prieto, R., Cuijpers, S. A., Luijsterburg, M. S., van Attikum, H., and Vertegaal, A. C. (2015). SUMOylation and PARylation cooperate to recruit and stabilize SLX4 at DNA damage sites. EMBO Rep. 16, 512-519. doi: 10.15252/embr.201440017

Gottschalk, A. J., Timinszky, G., Kong, S. E., Jin, J., Cai, Y., Swanson, S. K., et al. (2009). Poly(ADP-ribosyl)ation directs recruitment and activation of an ATP-dependent chromatin remodeler. Proc. Natl. Acad. Sci. U.S.A. 106, 13770-13774. doi: 10.1073/pnas.0906920106

Gudjonsson, T., Altmeyer, M., Savic, V., Toledo, L., Dinant, C., Grofte, M., et al. (2012). TRIP12 and UBR5 suppress spreading of chromatin ubiquitylation at damaged chromosomes. Cell 150, 697-709. doi: 10.1016/j.cell.2012.06.039

Guervilly, J. H., Takedachi, A., Naim, V., Scaglione, S., Chawhan, C., Lovera, Y., et al. (2015). The SLX4 complex is a SUMO E3 ligase that impacts on replication stress outcome and genome stability. Mol. Cell 57, 123-137. doi: 10.1016/j.molcel.2014.11.014

Haince, J. F., McDonald, D., Rodrigue, A., Dery, U., Masson, J. Y., Hendzel, M. J., et al. (2008). PARP1-dependent kinetics of recruitment of MRE11 and NBS1 proteins to multiple DNA damage sites. J. Biol. Chem. 283, 1197-1208. doi: 10.1074/jbc.M706734200

Hendriks, I. A., Treffers, L. W., Verlaan-de Vries, M., Olsen, J. V., and Vertegaal, A. C. (2015). SUMO-2 orchestrates chromatin modifiers in response to DNA damage. Cell Rep. doi: 10.1016/j.celrep.2015.02.033 [Epub ahead of print].

Hendzel, M. J., and Greenberg, R. A. (2013). Conversations between chromatin modifications and DNA double strand break repair: a commentary. Mutat. Res. 750, 1-4. doi: 10.1016/j.mrfmmm.2013.08.003

Ismail, I. H., Andrin, C., McDonald, D., and Hendzel, M. J. (2010). BMI1-mediated histone ubiquitylation promotes DNA double-strand break repair. J. Cell Biol. 191, 45-60. doi: 10.1083/jcb.201003034

Ismail, I. H., Gagne, J. P., Caron, M. C., McDonald, D., Xu, Z., Masson, J. Y., et al. (2012). CBX4-mediated SUMO modification regulates BMI1 recruitment at sites of DNA damage. Nucleic Acids Res. 40, 5497-5510. doi: 10.1093/nar/gks222

Ismail, I. H., Gagne, J. P., Genois, M. M., Strickfaden, H., McDonald, D., Xu, Z., et al. (2015). The RNF138 E3 ligase displaces Ku to promote DNA end resection and regulate DNA repair pathway choice. Nat. Cell Biol. 17, 1446-1457. doi: $10.1038 /$ ncb3259

Izhar, L., Adamson, B., Ciccia, A., Lewis, J., Pontano-Vaites, L., Leng, Y., et al. (2015). A systematic analysis of factors localized to damaged chromatin reveals PARP-dependent recruitment of transcription factors. Cell Rep 11, 1486-1500. doi: 10.1016/j.celrep.2015.04.053

Jackson, S. P., and Bartek, J. (2009). The DNA-damage response in human biology and disease. Nature 461, 1071-1078. doi: 10.1038/nature08467

Jackson, S. P., and Durocher, D. (2013). Regulation of DNA damage responses by ubiquitin and SUMO. Mol. Cell 49, 795-807. doi: 10.1016/j.molcel.2013. 01.017

Kang, H. C., Lee, Y. I., Shin, J. H., Andrabi, S. A., Chi, Z., Gagné, J. P., et al. (2011). Iduna is a poly(ADP-ribose) (PAR)-dependent E3 ubiquitin ligase that regulates DNA damage. Proc. Natl. Acad. Sci. U.S.A. 108, 14103-14108. doi: 10.1073/pnas. 1108799108

Kashima, L., Idogawa, M., Mita, H., Shitashige, M., Yamada, T., Ogi, K., et al. (2012). CHFR protein regulates mitotic checkpoint by targeting PARP-1 protein for ubiquitination and degradation. J. Biol. Chem. 287, 12975-12984. doi: 10.1074/jbc.M111.321828

Kim, H., and D'Andrea, A. D. (2012). Regulation of DNA cross-link repair by the Fanconi anemia/BRCA pathway. Genes Dev. 26, 1393-1408. doi: 10.1101/gad.195248.112

Krietsch, J., Rouleau, M., Pic, E., Ethier, C., Dawson, T. M., Dawson, V. L., et al. (2013). Reprogramming cellular events by poly(ADP-ribose)-binding proteins. Mol. Aspects Med. 34, 1066-1087. doi: 10.1016/j.mam.2012.12.005
Kuo, C. Y., Li, X., Stark, J. M., Shih, H. M., and Ann, D. K. (2016). RNF4 regulates DNA double-strand break repair in a cell cycle-dependent manner. Cell Cycle 15, 787-798. doi: 10.1080/15384101.2016.1138184

$\mathrm{Li}, \mathrm{M}$, and $\mathrm{Yu}, \mathrm{X}$. (2013). Function of BRCA1 in the DNA damage response is mediated by ADP-ribosylation. Cancer Cell 23, 693-704. doi: 10.1016/j.ccr.2013.03.025

Liu, C., Wu, J., Paudyal, S. C., You, Z., and Yu, X. (2013). CHFR is important for the first wave of ubiquitination at DNA damage sites. Nucleic Acids Res. 41, 1698-1710. doi: 10.1093/nar/gks1278

Lord, C. J., and Ashworth, A. (2016). BRCAness revisited. Nat. Rev. Cancer 16, 110-120. doi: 10.1038/nrc.2015.21

Lukas, J., Lukas, C., and Bartek, J. (2011). More than just a focus: the chromatin response to DNA damage and its role in genome integrity maintenance. Nat. Cell Biol. 13, 1161-1169. doi: 10.1038/ncb2344

Luo, K., Deng, M., Li, Y., Wu, C., Xu, Z., Yuan, J., et al. (2015). CDKmediated RNF4 phosphorylation regulates homologous recombination in S-phase. Nucleic Acids Res. 43, 5465-5475. doi: 10.1093/nar/gkv434

Luo, K., Zhang, H., Wang, L., Yuan, J., and Lou, Z. (2012). Sumoylation of MDC1 is important for proper DNA damage response. EMBO J. 31, 3008-3019. doi: 10.1038/emboj.2012.158

Mallette, F. A., Mattiroli, F., Cui, G., Young, L. C., Hendzel, M. J., Mer, G., et al. (2012). RNF8- and RNF168-dependent degradation of KDM4A/JMJD2A triggers 53BP1 recruitment to DNA damage sites. EMBO J. 31, 1865-1878. doi: 10.1038/emboj.2012.47

Marechal, A., and Zou, L. (2013). DNA damage sensing by the ATM and ATR kinases. Cold Spring Harb. Perspect. Biol. 5:a012716. doi: $10.1101 /$ cshperspect.a012716

Marteijn, J. A., Lans, H., Vermeulen, W., and Hoeijmakers, J. H. (2014). Understanding nucleotide excision repair and its roles in cancer and ageing. Nat. Rev. Mol. Cell Biol. 15, 465-481. doi: 10.1038/nrm3822

Martin, N., Schwamborn, K., Schreiber, V., Werner, A., Guillier, C., Zhang, X. D., et al. (2009). PARP-1 transcriptional activity is regulated by sumoylation upon heat shock. EMBO J. 28, 3534-3548. doi: 10.1038/emboj.2009.279

Mattiroli, F., Vissers, J. H., van Dijk, W. J., Ikpa, P., Citterio, E., Vermeulen, W., et al. (2012). RNF168 ubiquitinates K13-15 on H2A/H2AX to drive DNA damage signaling. Cell 150, 1182-1195. doi: 10.1016/j.cell.2012.08.005

Meerang, M., Ritz, D., Paliwal, S., Garajova, Z., Bosshard, M., Mailand, N., et al. (2011). The ubiquitin-selective segregase $\mathrm{VCP} / \mathrm{p} 97$ orchestrates the response to DNA double-strand breaks. Nat. Cell Biol. 13, 1376-1382. doi: 10.1038/ncb2367

Messick, T. E., and Greenberg, R. A. (2009). The ubiquitin landscape at DNA double-strand breaks. J. Cell Biol. 187, 319-326. doi: 10.1083/jcb.200908074

Messner, S., Schuermann, D., Altmeyer, M., Kassner, I., Schmidt, D., Schar, P., et al. (2009). Sumoylation of poly(ADP-ribose) polymerase 1 inhibits its acetylation and restrains transcriptional coactivator function. FASEB J. 23, 3978-3989. doi: 10.1096/fj.09-137695

Morris, J. R., Boutell, C., Keppler, M., Densham, R., Weekes, D., Alamshah, A., et al. (2009). The SUMO modification pathway is involved in the BRCA1 response to genotoxic stress. Nature 462, 886-890. doi: 10.1038/nature08593

Oberoi, J., Richards, M. W., Crumpler, S., Brown, N., Blagg, J., and Bayliss, R. (2010). Structural basis of poly(ADP-ribose) recognition by the multizinc binding domain of checkpoint with forkhead-associated and RING Domains (CHFR). J. Biol. Chem. 285, 39348-39358. doi: 10.1074/jbc.M110.159855

O'Connor, M. J. (2015). Targeting the DNA damage response in cancer. Mol. Cell 60, 547-560. doi: 10.1016/j.molcel.2015.10.040

Panier, S., and Boulton, S. J. (2014). Double-strand break repair: 53BP1 comes into focus. Nat. Rev. Mol. Cell Biol. 15, 7-18. doi: 10.1038/nrm3719

Panier, S., and Durocher, D. (2013). Push back to respond better: regulatory inhibition of the DNA double-strand break response. Nat. Rev. Mol. Cell Biol. 14, 661-672. doi: 10.1038/nrm3659

Patel, A., Lee, H. O., Jawerth, L., Maharana, S., Jahnel, M., Hein, M. Y., et al. (2015). A liquid-to-solid phase transition of the ALS protein FUS accelerated by disease mutation. Cell 162, 1066-1077. doi: 10.1016/j.cell.2015.07.047

Paull, T. T. (2015). Mechanisms of ATM activation. Annu. Rev. Biochem. 84, 711-738. doi: 10.1146/annurev-biochem-060614-034335

Pinder, J. B., Attwood, K. M., and Dellaire, G. (2013). Reading, writing, and repair: the role of ubiquitin and the ubiquitin-like proteins in DNA damage signaling and repair. Front. Genet. 4:45. doi: 10.3389/fgene.2013.00045 
Polo, S. E., and Jackson, S. P. (2011). Dynamics of DNA damage response proteins at DNA breaks: a focus on protein modifications. Genes Dev. 25, 409-433. doi: 10.1101/gad.2021311

Polo, S. E., Kaidi, A., Baskcomb, L., Galanty, Y., and Jackson, S. P. (2010). Regulation of DNA-damage responses and cell-cycle progression by the chromatin remodelling factor CHD4. EMBO J. 29, 3130-3139. doi: 10.1038/emboj.2010.188

Poulsen, S. L., Hansen, R. K., Wagner, S. A., van Cuijk, L., van Belle, G. J., Streicher, W., et al. (2013). RNF111/Arkadia is a SUMO-targeted ubiquitin ligase that facilitates the DNA damage response. J. Cell Biol. 201, 797-807. doi: 10.1083/jcb.201212075

Price, B. D., and D'Andrea, A. D. (2013). Chromatin remodeling at DNA doublestrand breaks. Cell 152, 1344-1354. doi: 10.1016/j.cell.2013.02.011

Ragland, R. L., Patel, S., Rivard, R. S., Smith, K., Peters, A. A., Bielinsky, A. K., et al. (2013). RNF4 and PLK1 are required for replication fork collapse in ATR-deficient cells. Genes Dev. 27, 2259-2273. doi: 10.1101/gad.223180.113

Rulten, S. L., Cortes-Ledesma, F., Guo, L., Iles, N. J., and Caldecott, K. W. (2008). APLF (C2orf13) is a novel component of poly(ADP-ribose) signaling in mammalian cells. Mol. Cell. Biol. 28, 4620-4628. doi: 10.1128/MCB.02243-07

Sarangi, P., Steinacher, R., Altmannova, V., Fu, Q., Paull, T. T., Krejci, L., et al. (2015). Sumoylation influences DNA break repair partly by increasing the solubility of a conserved end resection protein. PLoS Genet. 11:e1004899. doi: 10.1371/journal.pgen.1004899

Schmidt, C. K., Galanty, Y., Sczaniecka-Clift, M., Coates, J., Jhujh, S., Demir, M., et al. (2015). Systematic E2 screening reveals a UBE2D-RNF138-CtIP axis promoting DNA repair. Nat. Cell Biol. 17, 1458-1470. doi: 10.1038/ncb3260

Shiloh, Y., and Ziv, Y. (2013). The ATM protein kinase: regulating the cellular response to genotoxic stress, and more. Nat. Rev. Mol. Cell Biol. 14, 197-210. doi: $10.1038 / \mathrm{nrm} 3546$

Smeenk, G., Wiegant, W. W., Marteijn, J. A., Luijsterburg, M. S., Sroczynski, N., Costelloe, T., et al. (2013). Poly(ADP-ribosyl)ation links the chromatin remodeler SMARCA5/SNF2H to RNF168-dependent DNA damage signaling. J. Cell Sci. 126, 889-903. doi: 10.1242/jcs. 109413

Stracker, T. H., Usui, T., and Petrini, J. H. (2009). Taking the time to make important decisions: the checkpoint effector kinases Chk1 and Chk2 and the DNA damage response. DNA Repair (Amst.) 8, 1047-1054. doi: 10.1016/j.dnarep.2009.04.012

Teloni, F., and Altmeyer, M. (2016). Readers of poly(ADP-ribose): designed to be fit for purpose. Nucleic Acids Res. 44, 993-1006. doi: 10.1093/nar/gkv1383

Thorslund, T., Ripplinger, A., Hoffmann, S., Wild, T., Uckelmann, M., Villumsen, B., et al. (2015). Histone $\mathrm{H} 1$ couples initiation and amplification of ubiquitin signalling after DNA damage. Nature 527, 389-393. doi: $10.1038 /$ nature 15401

Timinszky, G., Till, S., Hassa, P. O., Hothorn, M., Kustatscher, G., Nijmeijer, B., et al. (2009). A macrodomain-containing histone rearranges chromatin upon sensing PARP1 activation. Nat. Struct. Mol. Biol. 16, 923-929. doi: 10.1038/nsmb.1664

Ulrich, H. D. (2014). Two-way communications between ubiquitin-like modifiers and DNA. Nat. Struct. Mol. Biol. 21, 317-324. doi: 10.1038/nsmb.2805

van Attikum, H., and Gasser, S. M. (2009). Crosstalk between histone modifications during the DNA damage response. Trends Cell Biol. 19, 207-217. doi: 10.1016/j.tcb.2009.03.001 van Cuijk, L., van Belle, G. J., Turkyilmaz, Y., Poulsen, S. L., Janssens, R. C., Theil, A. F., et al. (2015). SUMO and ubiquitin-dependent XPC exchange drives nucleotide excision repair. Nat. Commun. 6, 7499. doi: 10.1038/ncomms 8499

Wang, J., Aroumougame, A., Lobrich, M., Li, Y., Chen, D., Chen, J., et al. (2014). PTIP associates with Artemis to dictate DNA repair pathway choice. Genes Dev. 28, 2693-2698. doi: 10.1101/gad.252478.114

Wang, Z., Michaud, G. A., Cheng, Z., Zhang, Y., Hinds, T. R., Fan, E., et al. (2012). Recognition of the iso-ADP-ribose moiety in poly(ADP-ribose) by WWE domains suggests a general mechanism for poly(ADP-ribosyl)ationdependent ubiquitination. Genes Dev. 26, 235-240. doi: 10.1101/gad.182 618.111

Wu, J., Chen, Y., Lu, L. Y., Wu, Y., Paulsen, M. T., Ljungman, M., et al. (2011). Chfr and RNF8 synergistically regulate ATM activation. Nat. Struct. Mol. Biol. 18, 761-768. doi: $10.1038 / \mathrm{nsmb} .2078$

Xu, G., Chapman, J. R., Brandsma, I., Yuan, J., Mistrik, M., Bouwman, P., et al. (2015). REV7 counteracts DNA double-strand break resection and affects PARP inhibition. Nature 521, 541-544. doi: 10.1038/nature14328

Yan, Q., Xu, R., Zhu, L., Cheng, X., Wang, Z., Manis, J., et al. (2013). BAL1 and its partner E3 ligase, BBAP, link Poly(ADP-ribose) activation, ubiquitylation, and double-strand DNA repair independent of ATM, MDC1, and RNF8. Mol. Cell. Biol. 33, 845-857. doi: 10.1128/mcb.00990-12

Yin, Y. L., Seifert, A., Chua, J. S., Maure, J. F., Golebiowski, F., and Hay, R. T. (2012). SUMO-targeted ubiquitin E3 ligase RNF4 is required for the response of human cells to DNA damage. Genes Dev. 26, 1196-1208. doi: 10.1101/gad.189274.112

Zhang, F., Shi, J., Bian, C., and Yu, X. (2015). Poly(ADP-Ribose) mediates the BRCA2-dependent early DNA damage response. Cell Rep. 13, 678-689. doi: 10.1016/j.celrep.2015.09.040

Zhang, Y., Liu, S., Mickanin, C., Feng, Y., Charlat, O., Michaud, G. A., et al. (2011). RNF146 is a poly(ADP-ribose)-directed E3 ligase that regulates axin degradation and Wnt signalling. Nat. Cell Biol. 13, 623-629. doi: $10.1038 /$ ncb2222

Zhou, Z. D., Chan, C. H., Xiao, Z. C., and Tan, E. K. (2011). Ring finger protein 146/Iduna is a poly(ADP-ribose) polymer binding and PARsylation dependent E3 ubiquitin ligase. Cell Adh. Migr. 5, 463-471. doi: 10.4161/cam.5.6.18356

Zilio, N., Williamson, C. T., Eustermann, S., Shah, R., West, S. C., Neuhaus, D., et al. (2013). DNA-dependent SUMO modification of PARP-1. DNA Repair (Amst.) 12, 761-773. doi: 10.1016/j.dnarep.2013.07.001

Zimmermann, M., Lottersberger, F., Buonomo, S. B., Sfeir, A., and de Lange, T. (2013). 53BP1 regulates DSB repair using Rif1 to control 5' end resection. Science 339, 700-704. doi: 10.1126/science. 1231573

Conflict of Interest Statement: The authors declare that the research was conducted in the absence of any commercial or financial relationships that could be construed as a potential conflict of interest.

Copyright $\odot 2016$ Pellegrino and Altmeyer. This is an open-access article distributed under the terms of the Creative Commons Attribution License (CC BY). The use, distribution or reproduction in other forums is permitted, provided the original author(s) or licensor are credited and that the original publication in this journal is cited, in accordance with accepted academic practice. No use, distribution or reproduction is permitted which does not comply with these terms. 